\title{
Freezing DNA for Controlling Bio/nano Interfaces and Catalysis
}

\author{
Juewen Liu* \\ Department of Chemistry, Waterloo Institute for Nanotechnology, University of Waterloo, Waterloo, \\ Ontario, N2L 3G1, Canada
}

Email: liujw@uwaterloo.ca (J. L.)

\begin{abstract}
While most DNA-related reactions have been performed in solution, freezing has also been shown to offer interesting properties. DNA oligonucleotides are stretched and aligned in ice and the aligned DNA can quickly, tightly and in some cases densely adsorb on various nanomaterials. In addition, a few ribozymes and DNAzymes have better catalytic activities in ice than in solution. Some of these observations are related to the concentration of salt and DNA in the micropockets formed between ice crystals. With these interesting examples, future research can be devoted to detailed characterization of DNA in ice using in situ measurement methods, and finding their applications in biosensor development, catalysis and origin of life.
\end{abstract}

Keywords oligonucleotides, DNAzymes, bioconjugation, nanomaterials, adsorption

Over the last four decades, DNA oligonucleotides have become one of the most versatile molecules for a diverse range of applications from making sophisticated nanostructures, directed assembly of nanoparticles, biosensor development, to drug delivery. ${ }^{[1,2]}$ Such developments were enabled by solid phase DNA synthesis and by the excellent programmability, ligand binding and catalytic functions of DNA ${ }^{[3,4]}$ Most of DNA related reactions have been performed in solutions. Freezing is a condition used for long-term DNA storage, and chemical reactions are believed to be very slow at such low temperatures. Initial studies on DNA freezing was performed with long double-stranded DNA extracted from biological sources and these studied were focused on DNA preservation and damages.

When freezing takes place, ice crystals are formed. The non-water components are repelled by the crystals to pockets between them. ${ }^{[5]}$ These pockets between ice crystals are non-crystalline and the water with concentrated salt and other non-water species are in the eutectic phase. DNA is a polyelectrolyte with a very high density of negative charge. Many interesting observations have been made upon freezing DNA, which also showed useful applications.

An interesting recent observation made by us is that single-stranded DNA oligonucleotides of random sequences are aligned and stretched upon freezing. ${ }^{[6]}$ They form bundled structures enabled by base-base interactions, although such bundles may contain more than two DNA strands and typical base pairing interactions may not be required. We characterized this process by fluorescence resonance energy transfer using end-labeled DNA (Figure 1A). When dispersed in solution, the yellow emission was mainly from the FRET donor, Cy3 fluorophore. Aligned DNA increased intra-strand energy transfer showing a red emission upon freezing. This increase was inhibited by adding a large excess of non-labeled DNA of the same sequence, thus excluding the possibility of increased FRET due to intramolecular FRET. In addition, using thiazole orange dye for staining frozen DNA, we confirmed that DNA needs to be around 15-mer or longer to achieve optimal alignment, suggesting the importance of lateral base interactions. While a duplex DNA has a persistence length of around $50 \mathrm{~nm}$, the persistence length of single-stranded DNA is below $1 \mathrm{~nm}$. To stretch a single-stranded DNA often requires its hybridization with the complementary strand. Freezing provides a simple alternative method for this purpose.

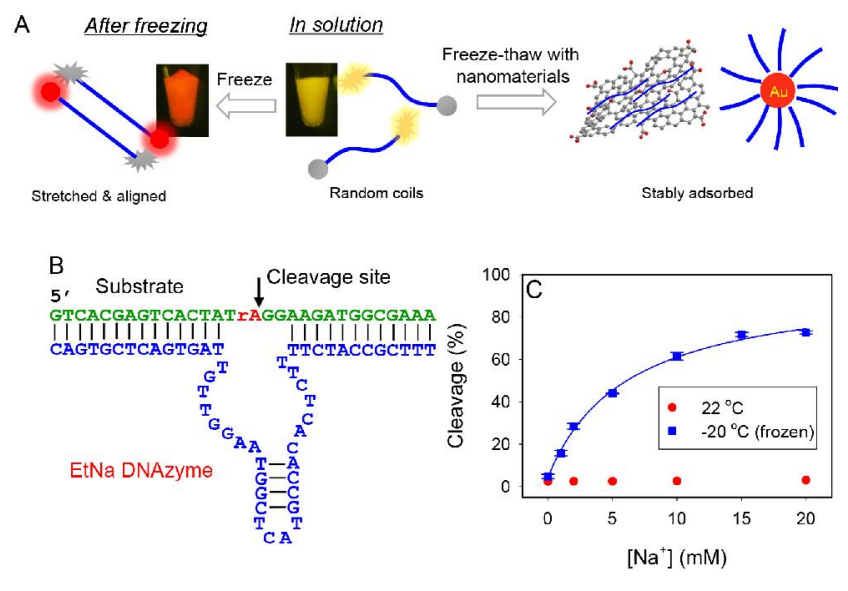

Figure 1 (A) Stretch and alignment of DNA oligonucleotides by freezing characterized using FRET. Aligned DNA increased inter-strand FRET. The aligned DNA is more stably adsorbed on graphene oxide and more densely adosrbed on AuNPs. Figure adapted from Ref. [6] with permission. Copyright John Wiley \& Sons 2019. (B) The secondary structure of a $\mathrm{Na}^{+}$dependent RNA-cleaving DNAzyme named EtNa. (C) With low $\mathrm{Na}^{+}$concentration, it is inactive in water but became active in ice. Figure adapted from Ref. [10] with permission. Copyright John Wiley \& Sons 2019.

It is common knowledge that nanoparticle dispersions cannot freeze, or they aggregate. Citrate-capped gold nanoparticles (AuNPs) are a representative example, and their aggregation is accompanied with a color change from red to blue. Interestingly, in the presence of a thiolated DNA, AuNPs can freeze and during the freezing process, the DNA molecules are densely attached (Figure 1A). ${ }^{[7]}$ Similar observations were also made with a few other nanomaterials such as metal oxide, graphene oxide and $\mathrm{MoS}_{2} \cdot{ }^{[6]}$ Compared to the attachment of DNA to these materials in solution, freezing produced more 


\section{Perspective}

stable conjugates, and this can be attributed to the stretched and aligned conformation of DNA upon freezing.

The above discussions used mainly the structural properties of DNA. In addition, DNA has also functional properties such as molecular recognition and catalysis. For example, DNA and RNA sequences with catalytic functions are called DNAzymes and ribozymes, respectively. Some ribozymes have been found in natural biological systems and many new ones have also been evolved in test tubes by scientists. Recently, their catalytic activities have also been pursued in ice. Holliger and coworkers performed in vitro selection of ribozymes with RNA polymerase activity that can work in ice. ${ }^{[8]}$ We previously isolated an RNA-cleaving DNAzyme named EtNa (Figure 1B), and found that its activity was activated by both ethanol, ${ }^{[9]}$ and by freezing. ${ }^{[10]}$ At low $\mathrm{Na}^{+}$ concentrations, no cleavage was observed in water, while efficient cleavage occurred in ice (Figure 1C). The mechanism of their acceleration could be from similar sources: increased electrostatic interactions with the salt. Upon freezing, $\mathrm{Na}^{+}$is concentrated near the DNAzyme and this can accelerate the reaction. Freezing does not stop the reaction and RNA cleavage continued for over a day at $-20{ }^{\circ} \mathrm{C}$ until saturated cleavage was achieved

The interest to study nucleic acid related reactions in ice has also been stimulated by curiosities on the origin of life. The ice and especially the sea ice environment is believed to be ideal for developing early forms of biochemical reactions ${ }^{[11]}$ For example, low temperature promotes DNA/RNA base pairing interactions, while high salt is useful to keep the primer and template strands together. Since replication of nucleic acids is key for the birth of life, extensive efforts have been made on this front. ${ }^{[12]}$ Given the interesting properties of ice, a few attempts have been made to elongate RNA with activated nucleotide imidazolides. ${ }^{[11,13,14]}$ In some cases, copying of a few hundred nucleotides was observed without the involvement of any enzymes, although the rates were quite slow. While these imidazole-activated monomers were unlikely to be used during evolution, they provide a faster reaction system for fundamental studies. Ribozymes have also been evolved for RNA replication in ice for offering insights into this front. ${ }^{[15]}$

As discussed above, interests on studying the property and reactions of nucleic acids in ice are for many different reasons. The origin of life community is mainly focused on RNA related reactions, while bio/nano researchers are more interested in DNA. With these interesting observations, we expect that the field experiences a lot of developments in the following directions. Firstly, fundamental studies are needed to understand the interaction between DNA with salt and comparison of DNA with other biopolymers and polymers during freezing. For fundamental understandings, in situ characterization of DNA during and after freezing is needed Fluorescence based methods are powerful and can give interesting information. Other methods based on electrons and high energy photons as probes are likely to provide complementary information. Introducing nanomaterials to the system is an important development. Aside from the benefits of improved DNA conjugation, nanomaterials provide various types of surfaces and they may also mimic the chemical systems related to the origin of life when water/ice is interfaced with minerals. In addition, by involving functional DNA such as aptamers and DNAzymes and understanding their reactions in the frozen state is interesting. Finally, analytical applications of these system will be pursued, in particular for cold regions where the sample could be frozen.

\section{References}

[1] Jones, M. R.; Seeman, N. C.; Mirkin, C. A. Science 2015, 347, 1260901.

[2] Zhou, W.; Saran, R.; Liu, J. Chem. Rev. 2017, 117, 8272.

[3] Tan, L. H.; Xing, H.; Lu, Y. Acc. Chem. Res. 2014, 47, 1881.

[4] Joyce, G. F. Ann. Rev. Biochem. 2004, 73, 791.

[5] O'Concubhair, R.; Sodeau, J. R. Acc. Chem. Res. 2013, 46, 2716.

[6] Liu, B.; Wu, T.; Huang, Z.; Liu, Y.; Liu, J. Angew. Chem. Int. Ed. 2019, 58, 2109.

[7] Liu, B.; Liu, J. J. Am. Chem. Soc. 2017, 139, 9471

[8] Attwater, J.; Wochner, A.; Holliger, P. Nat. Chem. 2013, 5, 1011.

[9] Zhou, W.; Saran, R.; Chen, Q.; Ding, J.; Liu, J. ChemBioChem 2016, 17, 159.

[10] Yu, T.; Zhou, W.; Liu, J. ChemBioChem 2018, 19, 1012.

[11] Trinks, H.; Schröder, W.; Biebricher, C. K. Origins Life Evol. B. 2005, 35, 429 .

[12] Mansy, S. S.; Schrum, J. P.; Krishnamurthy, M.; Tobe, S.; Treco, D. A.; Szostak, J. W. Nature 2008, 454, 122.

[13] Monnard, P.-A.; Szostak, J. W. J. Inorg. Biochem. 2008, 102, 1104.

[14] Monnard, P.-A.; Ziock, H. Chem. Biodivers. 2008, 5, 1521.

[15] Attwater, J.; Wochner, A.; Pinheiro, V. B.; Coulson, A.; Holliger, P. Nat. Commun. 2010, 1, 76 